Original Article

\title{
Lower limb kinematics of single-leg squat performance in patients with anterior cruciate ligament deficiency
}

\author{
Shigeki Yokoyama, RPT, PhD ${ }^{1)^{*}}$, Wataru Fukuda, RPT, $\mathrm{MS}^{2)}$, Yutaro IKeno, RPT ${ }^{2)}$, \\ Yusuke Kataoka, RPT ${ }^{2)}$, SEAN A. Horan, RPT, PhD ${ }^{3)}$ \\ 1) Kyoto Tachibana University: 34 Yamada-cho, Oyake, Yamashina-ku, Kyoto 607-8175, Japan \\ 2) Department of Physical Therapy, Center of Joint Surgery, Kaisei General Hospital, Japan \\ 3) School of Allied Health Sciences, Griffith University, Australia
}

\begin{abstract}
Purpose] This study investigated the parameters that characterize the knee, hip, and pelvic kinematics during a single-leg squat in preoperative anterior cruciate ligament rupture injury. [Participants and Methods] Overall, 15 patients with unilateral anterior cruciate ligament deficiency were enrolled in this study. For each single-leg squat, data from two-dimensional video cameras and three-dimensional motion analysis were collected. Measurement indices included the articular angles of the knee, hip, and trunk. The anterior cruciate ligament-injured leg was compared with the uninjured leg. [Results] The maximum knee valgus and flexion angles during a single-leg squat were smaller in the injured leg than in the uninjured leg. During the single-leg squat, the effect of "compensatory mechanisms" appeared as knee valgus and flexion movements. In particular, the knee valgus angle decreased in the anterior cruciate ligament-injured leg compared to that in the uninjured leg. [Conclusion] This phenomenon suggests that it is possible to utilize recurrence prevention training for anterior cruciate ligament injury. Key words: Knee dynamic valgus, Three-dimensional kinematic analysis, Compensatory mechanisms
\end{abstract}

(This article was submitted Jan. 10, 2021, and was accepted Feb. 23, 2021)

\section{INTRODUCTION}

Anterior cruciate ligament (ACL) injuries are among the most debilitating sports injuries suffered by amateur and professional athletes ${ }^{1,2)}$. Young athletes are particularly susceptible to non-contact ACL injuries ${ }^{2-4)}$. Individuals with ACL injuries require long recovery time, with most individuals not returning to sports for 9-12 months ${ }^{5)}$. During the recovery period, which in most cases involves surgical repair or reconstruction of the ACL, individuals will undertake several hours of rehabilitation usually under the direction of a physical therapist. To determine the most appropriate rehabilitation strategies for a patient with an ACL injury, it is crucial to modify the program based on their individual needs. For the physical therapist, this is typically achieved by performing a thorough physical examination (often pre-operatively), which assists in establishing key outcome measures as well as guiding the development of the patients' exercise and rehabilitation program.

The single-leg squat (SLS) test is a physical examination used by many physical therapists for the assessment of patients with lower limb injury, including those with suspected ACL injuries. The test provides information about a patient's confidence and stability on one leg, as well as information about knee pain and a patient's pattern of movement that might be considered undesirable or potentially injurious ${ }^{6,7)}$. The dynamic valgus position of the knee during single-leg activities is one movement pattern that has received much attention in the literature and has been associated with higher rates of knee injuries $^{8,9)}$. Other abnormal movement patterns observed during an SLS include excessive trunk or pelvis motion, general unsteadiness or balance difficulties, and lack of squat depth. If such movement patterns are identified in a patient, a physical

*Corresponding author. Shigeki Yokoyama (E-mail: yokoyama-s@tachibana-u.ac.jp)

(C2021 The Society of Physical Therapy Science. Published by IPEC Inc.

(c) $(-)$ This is an open-access article distributed under the terms of the Creative Commons Attribution Non-Commercial No DerivaCC BY NC ND tives (by-nc-nd) License. (CC-BY-NC-ND 4.0: https://creativecommons.org/licenses/by-nc-nd/4.0/) 
therapist can provide targeted treatment strategies early in the rehabilitation process. This is particularly important in patients who demonstrate dynamic knee valgus because aberrant movement patterns can place significant stress on a normal healthy ACL, not to mention a newly repaired one ${ }^{10}$. Furthermore, changing a patient's "pattern of movement" can be challenging; therefore, early input is critical for achieving better long-term movement patterns.

To date, few studies have examined pre-operative lower limb kinematics during an SLS in patients with both a healthy and an ACL-deficient knee. We were, therefore, interested in determining if side-to-side differences in knee, hip, and pelvis kinematics existed during SLS for pre-operative ACL-injured patients. We hypothesized that ACL-injured patients would demonstrate a larger dynamic knee valgus angle as well as a decreased depth of SLS for the injured leg compared to the uninjured leg.

\section{PARTICIPANTS AND METHODS}

Fifteen patients with unilateral ACL deficiency volunteered to participate in the study (10 males and 5 females). The inclusion criteria consisted of participants being able to walk unaided, perform their normal activities of daily living without assistance, a positive Lachman's test, and excessive anterior tibial translation $(>4 \mathrm{~mm})$ measured using instrumented arthrometry (KT1000; MED Metric Corporation, San Diego, CA, USA). Both the Lachman's test and instrumented tibial translation test were performed by the same orthopedic surgeon (Kaisei Hospital, Kagawa, Japan). Potential participants were excluded if they had a current or previous medical, musculoskeletal (e.g., low back pain), or neurological condition (e.g., concussion) that impaired their ability to perform an SLS.

Participants were recruited via word-of-mouth at Kaisei Hospital where they provided written informed consent to participate. All participants visited the orthopedic surgeon and physical therapist on at least one occasion where they were provided with a home exercise program that included knee range-of-motion exercises as well as quadriceps and hamstring strengthening exercises. No double- or single-leg squatting exercises were included in the home exercise program. The study was approved by the Kaisei Hospital's ethics committee (approval number, 2008-6).

Participants were required to wear sports shorts and a singlet and to remove any footwear during the SLS testing. All participants performed SLS testing on both legs starting with the uninjured leg followed by the injured leg. Standard instructions were given to all participants, which included standing on one leg with their arms folded, placing their non-stance leg behind them, and looking straight ahead before flexing their knee in a slow controlled manner (i.e., approximately 1 squat per 2 seconds). To facilitate participant understanding, one of the investigators demonstrated the performance of an SLS for all participants. Each participant was advised to maintain their balance and not lean forward while attempting to squat down to a depth of 60 degrees of knee flexion. Prior to testing, all participants were required to perform a minimum of three consecutive SLS practice attempts where they achieved a depth of 60 degrees of knee flexion, as measured using a goniometer. Once successful, the participants performed three test trials. The same procedure was followed for the uninjured and injured legs.

Two-dimensional (2D) marker data were collected using four digital video cameras at 30 frames per second (DCRTRV17 K, Sony Corporation, Tokyo, Japan) in conjunction with TOMOCO-VM 3D analysis software (Toso System, Ageo, Japan). Each camera was placed on a tripod, approximately $4 \mathrm{~m}$ away from the participant, diagonally to their line of direction and at a height of approximately $90 \mathrm{~cm}$. Prior to the collection of experimental data, a number of calibration trials were recorded by positioning a calibration frame $(2 \mathrm{~m} \times 1.8 \mathrm{~m})$ in the capture volume, which subsequently enabled direct linear transformation of 2D marker data to 3D marker data. Color markers $(20 \mathrm{~mm})$ were attached to specific anatomic landmarks including the right and left lateral malleolus, lateral femoral condyle, greater trochanter, and acromial process.

Transformed 3D coordinate data were then used for computing projection angles of the hip and knee joints and pelvis segment. Specifically, the hip flexion-extension angle was calculated as the angle between the line connecting the lateral femoral condyle and greater trochanter and the line connecting the greater trochanter and acromion process projected onto the sagittal plane. The hip abduction-adduction angle was calculated as the angle between the same lines used for calculating the hip flexion-extension angle except projected onto the frontal plane. The knee flexion-extension angle was calculated as the angle between the line connecting the lateral malleolus and femoral condyle and the line connecting the lateral femoral condyle and greater trochanter projected onto the sagittal plane. The knee varus-valgus angle was obtained as the angle between the same lines used for calculating the knee flexion-extension except that it is projected onto the frontal plane. The pelvic obliquity angle (i.e., lateral tilt angle) was calculated as the angle between the line connecting the right and left greater trochanter and the line connecting the lateral femoral condyle and greater trochanter of the support leg projected onto the frontal plane. The pelvic obliquity angle was considered positive when elevation of the pelvic line occurred on the supporting leg.

Following calculations of the joint and segment angles, angular data at discrete time points were used for the comparison of the ACL-injured leg with the uninjured leg. For the hip joint, the data included the maximum flexion and extension angles, maximum abduction and adduction angles, and abduction angle at the point of maximum SLS depth (i.e., the point of maximum knee flexion). For the knee joint, the maximum flexion and extension angles were determined as well as the maximum valgus and varus angles and valgus angle at the point of maximum SLS depth. For the pelvis, the maximum (xii) obliquity angle was determined during SLS.

All the data are presented as mean \pm standard deviation. The data were checked and confirmed to have normal distribution using the Shapiro-Wilk verification. The Wilcoxon signed-rank test was used for evaluating each measurement index 
between the injured and uninjured legs. The Pearson correlations and Spearman rank correlation coefficients were used for determining the relationship between each discrete kinematic variable. All statistical analyses were performed using SPSS for Windows, Version 22.0 (IBM, Chicago, IL, USA), with significance levels set a priori at $\alpha=0.05$.

\section{RESULTS}

A total of 15 patients ( 10 males and 5 females) with a mean age of $24.2 \pm 6.5$ years participated in the study. Participants were $169.2 \pm 9.4 \mathrm{~cm}$ tall, with a body mass of $67.2 \pm 13.3 \mathrm{~kg}$ and a body mass index (BMI) of $23.3 \pm 2.9$. Among the 15 participants, 11 had non-contact injuries, and 4 had contact injuries. The mean duration between the time of initial injury and SLS assessment was $85.0 \pm 52.6$ days.

Descriptive statistics for the kinematic variables of interest are presented in Table 1. During the experimental trials, no statistically significant difference in the maximum hip flexion or knee flexion between the injured and uninjured leg was observed; however, a trend for decreased knee flexion for the injured leg during SLS was evident. The maximum knee valgus angle was significantly smaller for the injured leg than for the uninjured leg $\left(9.7^{\circ} \pm 5.2^{\circ}\right.$ vs. $\left.15.2^{\circ} \pm 8.0^{\circ}, p=0.027\right)$. Similarly, at the point of maximum SLS depth, the knee valgus angle was significantly smaller for the injured leg than for the uninjured $\operatorname{leg}\left(7.3^{\circ} \pm 5.5^{\circ}\right.$ vs. $\left.13.0^{\circ} \pm 8.5^{\circ} ; \mathrm{p}=0.031\right)$.

The Pearson product-moment correlations were determined for examining the relationships between the discrete kinematic variables of interest (Table 2). For the knee joint, a significant positive correlation $(r=0.58, p=0.019)$ was observed between the maximum knee flexion and knee valgus angles for the injured leg. Similarly, a significant positive correlation $(\mathrm{r}=0.50, \mathrm{p}=0.047)$ was observed between the maximum knee flexion and knee valgus angles at the maximum squat depth for the injured leg. No significant correlations for the knee joint were observed for the uninjured group. For the hip joint, a significant negative correlation $(\mathrm{r}=-0.97$; $\mathrm{p}<0.001)$ was observed between the maximum hip abduction and knee valgus angles for the injured leg. No significant correlations for the hip joint were observed for the uninjured leg. Furthermore, no significant correlations for the pelvis segment were observed for either the injured or the uninjured legs.

Table 1. Maximum joint angle during a single-leg squat

\begin{tabular}{llccc}
\hline Region & Direction & Injured $\left(^{\circ}\right)$ & Uninjured $\left({ }^{\circ}\right)$ & Cohen's d \\
\hline Trunk & Lateral tilt (upward + ) & $9.0 \pm 7.6$ & $6.2 \pm 4.7$ & 0.44 \\
\hline \multirow{4}{*}{ Hip } & Flexion & $42.0 \pm 15.4$ & $40.3 \pm 14.6$ & 0.11 \\
& Extension & $0.1 \pm 7.0$ & $1.8 \pm 7.5$ & 0.23 \\
& Abduction & $8.6 \pm 10.1$ & $6.4 \pm 9.1$ & 0.23 \\
& Abduction (Knee flex max.) & $-2.6 \pm 16.3$ & $3.3 \pm 16.2$ & 0.36 \\
& Adduction & $5.9 \pm 10.0$ & $10.6 \pm 9.9$ & 0.47 \\
\hline \multirow{6}{*}{ Knee } & Flexion & $52.8 \pm 9.5$ & $57.0 \pm 10.0$ & 0.43 \\
& Extension & $-3.2 \pm 5.0$ & $0.2 \pm 7.4$ & 0.54 \\
& Valgus* & $9.7 \pm 5.2$ & $15.2 \pm 8.0$ & 0.82 \\
& Valgus* (Knee flex max.) & $7.3 \pm 5.5$ & $13.0 \pm 8.5$ & 0.80 \\
& Varus & $3.0 \pm 3.9$ & $3.1 \pm 4.0$ & 0.03 \\
\hline
\end{tabular}

Mean \pm SD. ${ }^{*} \mathrm{p} \leq 0.05$ indicates a significant difference between the injured and uninjured knee.

Table 2. Correlation coefficients between the kinematics of the knee and each region

\begin{tabular}{lllccc}
\hline \multirow{2}{*}{ Region } & Direction & Side & Knee flexion & Knee valgus & $\begin{array}{c}\text { Knee valgus } \\
\text { (flex-max) }\end{array}$ \\
\hline \multirow{2}{*}{ Trunk } & Lateral tilt & Injured & -0.42 & -0.17 & -0.14 \\
& (upward +) & Uninjured & -0.41 & 0.08 & 0.08 \\
\hline \multirow{2}{*}{ Hip } & Flexion & Injured & 0.37 & 0.19 & 0.13 \\
& & Uninjured & $0.52^{*}$ & 0.19 & 0.07 \\
\cline { 3 - 6 } & Abduction & Injured & -0.20 & -0.03 & $-0.97^{*}$ \\
& & Uninjured & -0.16 & -0.26 & -0.26 \\
\cline { 3 - 6 } & Abduction & Injured & -0.10 & 0.10 & 0.10 \\
& (Knee flex max) & Uninjured & -0.02 & -0.01 & -0.03 \\
\hline \multirow{2}{*}{ Knee } & Flexion & Injured & - & $0.58^{*}$ & $0.50^{*}$ \\
& & Uninjured & - & 0.06 & -0.01 \\
\hline \multirow{2}{*}{ Cor } & & & & &
\end{tabular}

*Correlations had a significant value at $\mathrm{p}<0.05$ level. 


\section{DISCUSSION}

We observed a larger knee valgus angle for the uninjured leg than for the injured leg during SLS. While not statistically significant, we also observed a trend toward decreased SLS depth for the injured leg, as evidenced by a smaller knee flexion angle.

In partial agreement with our hypotheses, patients demonstrated decreased knee flexion for the uninjured leg compared with that of the injured leg during SLS testing. However, in disagreement with our original hypotheses, patients demonstrated a smaller maximum knee valgus angle and a smaller knee valgus angle at maximum SLS depth for the uninjured leg than for the injured leg. This finding suggests that the dynamic knee valgus angle was smaller on the side of ACL injury during SLS, which at first examination was somewhat counterintuitive. However, it is plausible that this was a compensatory movement adopted by patients for decreasing the mechanical load on the injured knee; patients with a ruptured ACL may subconsciously avoid a dynamic valgus position as a protective movement pattern. Moreover, patients with a ruptured ACL are likely to be apprehensive about performing an SLS in the greater degrees of knee flexion, as it is likely to feel unstable and will require greater muscular force and control from the quadriceps and hip abductor muscle groups ${ }^{11,12}$. Generating sufficient forces in these muscle groups are likely to be challenging owing to muscle inhibition, which is often associated with an acute ligament injury; weakness and deconditioning are, in addition, common consequences of disuse or unloading ${ }^{13,14)}$. In agreement with our findings, Yamazaki and colleagues ${ }^{15}$ ) reported kinematic differences between the injured and uninjured leg in a group of patients with a unilateral ACL injury. Specifically, both ACL-injured males and females did not flex their injured knee to the same depth as their uninjured knee during SLS testing; however, at odds with our findings, patients in the Yamazaki study demonstrated a larger varus angle for the injured leg than for the uninjured leg. Bulgheroni et al. ${ }^{16)}$ observed a phenomenon called "compensatory mechanisms" wherein the muscular activities of the quadriceps decreased and that of the hamstrings increased during gait training; therefore, they reported that the flexion movement of the knee joint in the sagittal plane decreased with a change in muscle activity during knee walking. During a squat motion, the knee valgus movement may also have been controlled in the frontal plane. Indeed, it is thought that compensatory muscle contractions during a squat movement are effective from the viewpoint of preventing the recurrence of an ACL injury. Interestingly, in our study, the phenomenon of compensatory movement appeared in the frontal plane during the squat movement, suggesting this movement may be useful for rectifying dynamic alignment of knee valgus in postoperative physiotherapy.

When the knee flexion angle was large, a tendency of the valgus angle to also be large was observed for the ACL-injured knee during the squat motion; however, the knee flexion angle and the valgus angle were unrelated in the non-injured knee. Murayama et al. ${ }^{17}$ ) reported that the movement range in the anteroposterior direction for the medial femoral condyle was larger for the ACL-injured leg than the uninjured leg during an SLS. The observation that the varus angle increases with knee flexion in an ACL injured leg during an SLS is regarded as anterior-medial instability owing to ACL dysfunction.

There were some limitations to this study. First, SLS testing tends to have a greater influence on the muscular strength and balance ability than that of double-leg squats. However, this was not measured and verified in our study. Second, there was a combination of male and female patients with ACL injuries who participated in this study. In the precedent study, there were gender-related differences in the joint movements during SLS ${ }^{18,19)}$. However, we did not perform a comparison between men and women due to the small sample size in this study. Nevertheless, there is a possibility of gender-related differences in terms of kinematics in the injured leg of male individuals versus that of female individuals. Therefore, it is necessary to verify this matter in future studies.

In conclusion, the influence of compensatory mechanisms on the ACL-injured knee during an SLS was observed in the frontal plane. Particularly, it is important for the phenomenon in which the knee valgus for the ACL-injured side becomes smaller than that for the uninjured side from the viewpoint of recrudescence prevention. As such, this phenomenon may be applied to a motor learning program for modifying dynamic alignment to reduce recurrence risk after an ACL injury.

Funding and Conflict of interest

None.

\section{REFERENCES}

1) de Loës M, Dahlstedt LJ, Thomée R: A 7-year study on risks and costs of knee injuries in male and female youth participants in 12 sports. Scand J Med Sci Sports, 2000, 10: 90-97. [Medline] [CrossRef]

2) Ardern CL, Webster KE, Taylor NF, et al.: Return to sport following anterior cruciate ligament reconstruction surgery: a systematic review and meta-analysis of the state of play. Br J Sports Med, 2011, 45: 596-606. [Medline] [CrossRef]

3) Agel J, Arendt EA, Bershadsky B: Anterior cruciate ligament injury in national collegiate athletic association basketball and soccer: a 13-year review. Am J Sports Med, 2005, 33: 524-530. [Medline] [CrossRef]

4) Frobell RB, Roos EM, Roos HP, et al.: A randomized trial of treatment for acute anterior cruciate ligament tears. N Engl J Med, 2010, 363: 331-342. [Medline] [CrossRef] 
5) Lai CC, Ardern CL, Feller JA, et al.: Eighty-three per cent of elite athletes return to preinjury sport after anterior cruciate ligament reconstruction: a systematic review with meta-analysis of return to sport rates, graft rupture rates and performance outcomes. Br J Sports Med, 2018, 52: 128-138. [Medline] [CrossRef]

6) Nae J, Creaby MW, Nilsson G, et al.: Measurement properties of a test battery to assess postural orientation during functional tasks in patients undergoing anterior cruciate ligament injury rehabilitation. J Orthop Sports Phys Ther, 2017, 47: 863-873. [Medline]

7) Trulsson A, Miller M, Gummesson C, et al.: Associations between altered movement patterns during single-leg squat and muscle activity at weight-transfer initiation in individuals with anterior cruciate ligament injury. BMJ Open Sport Exerc Med, 2017, 2: e000131. [Medline] [CrossRef]

8) Kobayashi H, Kanamura T, Koshida S, et al.: Mechanisms of the anterior cruciate ligament injury in sports activities: a twenty-year clinical research of 1,700 athletes. J Sports Sci Med, 2010, 9: 669-675. [Medline]

9) Hewett TE, Myer GD, Ford KR, et al.: Biomechanical measures of neuromuscular control and valgus loading of the knee predict anterior cruciate ligament injury risk in female athletes: a prospective study. Am J Sports Med, 2005, 33: 492-501. [Medline] [CrossRef]

10) Markolf KL, Burchfield DM, Shapiro MM, et al.: Combined knee loading states that generate high anterior cruciate ligament forces. J Orthop Res, 1995, 13: 930-935. [Medline] [CrossRef]

11) Thambyah A, Thiagarajan P, Goh Cho Hong J: Knee joint moments during stair climbing of patients with anterior cruciate ligament deficiency. Clin Biomech (Bristol, Avon), 2004, 19: 489-496. [Medline] [CrossRef]

12) Stickler L, Finley M, Gulgin H: Relationship between hip and core strength and frontal plane alignment during a single leg squat. Phys Ther Sport, 2015, 16: 66-71. [Medline] [CrossRef]

13) Frank RM, Lundberg H, Wimmer MA, et al.: Hamstring activity in the anterior cruciate ligament injured patient: injury implications and comparison with quadriceps activity. Arthroscopy, 2016, 32: 1651-1659. [Medline] [CrossRef]

14) Shanbehzadeh S, Mohseni Bandpei MA, Ehsani F: Knee muscle activity during gait in patients with anterior cruciate ligament injury: a systematic review of electromyographic studies. Knee Surg Sports Traumatol Arthrosc, 2017, 25: 1432-1442. [Medline] [CrossRef]

15) Yamazaki J, Muneta T, Ju YJ, et al.: Differences in kinematics of single leg squatting between anterior cruciate ligament-injured patients and healthy controls. Knee Surg Sports Traumatol Arthrosc, 2010, 18: 56-63. [Medline] [CrossRef]

16) Bulgheroni P, Bulgheroni MV, Andrini L, et al.: Walking in anterior cruciate ligament injuries. Knee, 1997, 4: 159-165. [CrossRef]

17) Murayama $T$, Sato $T$, Watanabe $S$, et al.: Three-dimensional in vivo dynamic motion analysis of anterior cruciate ligament-deficient knees during squatting using geometric center axis of the femur. J Orthop Sci, 2016, 21: 159-165. [Medline] [CrossRef]

18) Numata H, Nakase J, Kitaoka K, et al.: Two-dimensional motion analysis of dynamic knee valgus identifies female high school athletes at risk of non-contact anterior cruciate ligament injury. Knee Surg Sports Traumatol Arthrosc, 2018, 26: 442-447. [Medline] [CrossRef]

19) Gwinn DE, Wilckens JH, McDevitt ER, et al.: The relative incidence of anterior cruciate ligament injury in men and women at the United States Naval Academy. Am J Sports Med, 2000, 28: 98-102. [Medline] [CrossRef] 